\title{
Publisher Correction: Rapid desolvation-triggered domino lattice rearrangement in a metal-organic framework
}

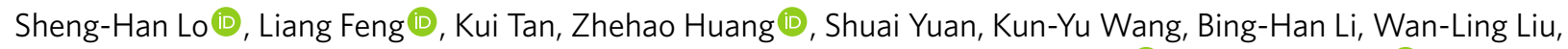
Gregory S. Day, Songsheng Tao, Chun-Chuen Yang, Tzuoo-Tsair Luo, Chia-Her Lin $\mathbb{1}$, Sue-Lein Wang $\mathbb{B}$,

Simon J. L. Billinge $\mathbb{D}$, Kuang-Lieh Lu $\mathbb{D}^{\mathbb{B}}$, Yves J. Chabal, Xiaodong Zou and Hong-Cai Zhou $\mathbb{D}$

Correction to: Nature Chemistry https://doi.org/10.1038/s41557-019-0364-0, published online 2 December 2019.

In the version of this Article originally published, affiliations 8 and 9 were incorrectly switched. Affiliation 8 was listed as 'Department of Chemistry, Chung-Yuan Christian University, Taoyuan City, Taiwan', but should have been 'Department of Physics, Chung-Yuan Christian University, Taoyuan City, Taiwan', and vice versa for affiliation 9. This has now been corrected.

Published online: 10 December 2019

https://doi.org/10.1038/s41557-019-0404-9

(๑) The Author(s), under exclusive licence to Springer Nature Limited 2019 